\title{
SPACE-TIME CROSS CORRELATION AND SPACE-FREQUENCY CROSS SPECTRUM IN NON-ISOTROPIC SCATTERING ENVIRONMENTS
}

\author{
T. A. Lamahewa, T. D. Abhayapala* ,R. A. Kennedy* \\ Department of Information Engineering (RSISE), \\ The Australian National University, Canberra, \\ ACT 0200, Australia.
}

\author{
J. T. Y. Ho
}

\author{
Department of Electrical and \\ Computer Systems Engineering \\ Building 72, Monash University, \\ VIC 3800, Australia.
}

\begin{abstract}
This paper presents a method which allows to obtain expressions for the space-time cross correlation (STCC) and the space-frequency cross spectrum (SFCS) between signals at two receiver antennas on a mobile unit (MU) for an arbitrary scattering distribution surrounding the MU.
\end{abstract}

\section{INTRODUCTION}

Space-time wireless channel modeling has received attention in recent years [1-3] since space-time processing using multiple antennas is becoming one of the most promising areas for the improvement of the performance of mobile communication systems. In channel modeling research, the effects of spatial and temporal correlation are of primary importance as they impact the performance of space-time communication systems.

A SIMO space-time channel model has been proposed in [1] based on the Clarke's classic model, assuming scatterers around the MU antenna are uniformly distributed on a ring, but the base station (BS) antennas are absente of local scatterers. Corresponding spacetime cross correlation and space-frequency cross spectrum functions between two antennas at the BS were also derived. In [2], these functions were generalized by incorporating a non-isotropic distribution of scatterers around the MU and the model has been extended to the case of a MIMO channel where the receiver antennas are assumed to be co-located. A general space-time cross correlation function for a MIMO fading channel was proposed in [3], assuming a nonisotropic scattering distribution around the MU antennas with the scatterers located on a ring. An expression for the space-time cross correlation is derived for a von-Mises distributed field [4] around the MU.

In this paper, we obtain expressions for the space-time cross correlation and the space-frequency cross spectrum between signals at two receiver antennas on a MU for any general scattering distribution. The presented method follows from the plane-wave modal expansion technique used in [5] to obtain spatial correlation between two receiver antenna elements. The method is illustrated for an unimodal Laplacian distribution, where the distribution is restricted to a

${ }^{*}$ T.D. Abhayapala and R.A. Kennedy are also with National ICT Australia, Locked Bag 8001, Canberra, ACT 2601, AUSTRALIA. National ICT Australia is funded through the Australian Government's Backing Australia's Ability initiative, in part through the Australian Research Council. This work was supported by the Australian Research Council Discovery Grant DP0343804. limited spread, and a multi-modal distribution (with von-Mises components), but the method is equally applicable for a multitude of scattering distributions.

Notations: Throughout the paper, the following notations will be used: $(\cdot)^{*}$ denotes the complex conjugate operation. The symbol $\delta(\cdot)$ denotes the Dirac delta function and $i=\sqrt{-1}$. The notation $E\{\cdot\}$ denotes the mathematical expectation, $\lceil$.$\rceil denotes the ceiling$ operator and $\|\cdot\|$ denotes the Euclidean length of a vector. Bold lower letters denote vectors and $\hat{\boldsymbol{x}}$ denotes a unit vector.

\section{SPACE-TIME CROSS CORRELATION}

Consider two receiver antennas located on a MU at points $\boldsymbol{x}_{1}$ and $\boldsymbol{x}_{2}$ at time $t=0$. Let $s_{1}(t)$ and $s_{2}(t)$ denote the received signal at two antennas and assume that they are wide-sense stationary. Then the normalized space-time cross correlation function between the complex envelopes of $s_{1}(t)$ and $s_{2}(t)$ is defined by

$$
R_{1,2}(\tau)=\frac{E\left\{s_{1}(t) s_{2}^{*}(t-\tau)\right\}}{\sqrt{E\left\{\left|s_{1}(t)\right|^{2}\right\} E\left\{\left|s_{2}(t-\tau)\right|^{2}\right\}}} .
$$

We consider a general scattering environment with scatterers distributed in the far field from the receiver antennas. If the MU is moving at constant velocity $v$ in the direction $\hat{\boldsymbol{v}}$ and the transmitted signal is a narrowband signal $e^{i \omega_{c} t}$, where $\omega_{c}$ is the angular carrier frequency of the signal, then the narrowband signal impinging on the $m^{\text {th }}$ receiver at point $\boldsymbol{x}_{m}$ is

$$
\begin{aligned}
s_{m}(t) & =\int_{\Omega} a(\hat{\boldsymbol{\phi}}) e^{i\left(\omega_{c}+\omega_{d}(\hat{\boldsymbol{\phi}})\right) t} e^{-i k \boldsymbol{x}_{m} \cdot \hat{\boldsymbol{\phi}}} d \hat{\boldsymbol{\phi}}, \\
& =e^{i \omega_{c} t} \int_{\Omega} a(\hat{\boldsymbol{\phi}}) e^{-i k \boldsymbol{u}_{m}(t) \cdot \hat{\boldsymbol{\phi}}} d \hat{\boldsymbol{\phi}}
\end{aligned}
$$

where $\omega_{d}(\hat{\boldsymbol{\phi}})=\frac{2 \pi v}{\lambda} \hat{\boldsymbol{v}} \cdot \hat{\boldsymbol{\phi}}$ is the angular Doppler spread, $\boldsymbol{u}_{m}(t)=$ $\boldsymbol{x}_{m}-t v \hat{\boldsymbol{v}}$ is the position of the $m^{\text {th }}$ receiver antenna at time $t, k=$ $2 \pi / \lambda$ is the wave number with $\lambda$ the wave length, $\hat{\phi}$ is a unit vector pointing in the direction of wave propagation, $a(\hat{\phi})$ is the effective random complex gain of scatterers for the transmitted signal arriving at the receiver from direction $\hat{\phi}$. Note that the integration in (2) is over a unit sphere in a 3-D multipath field or over a unit circle in a 2-D multipath field.

Assume that the scattering from one direction is independent of that from another direction at the receiver antennas. Then we have

$$
E\left\{a(\hat{\phi}) a^{*}\left(\hat{\phi}^{\prime}\right)\right\}=A(\hat{\phi}) \delta\left(\hat{\phi}-\hat{\phi}^{\prime}\right),
$$


where $A(\hat{\phi})=E\left\{|a(\hat{\phi})|^{2}\right\}$. Substituting (2) in (1) and using (3), we get

$$
R_{1,2}(\tau)=e^{i \omega_{c} \tau} \int_{\Omega} \mathcal{P}(\hat{\boldsymbol{\phi}}) e^{i k \boldsymbol{u}(\tau) \cdot \hat{\boldsymbol{\phi}}} d \hat{\boldsymbol{\phi}},
$$

where $\boldsymbol{u}(\tau)=\boldsymbol{u}_{2}(t-\tau)-\boldsymbol{u}_{1}(t)=\boldsymbol{x}_{2}-\boldsymbol{x}_{1}+\tau v \hat{\boldsymbol{v}}$ and

$$
\mathcal{P}(\hat{\phi})=\frac{E\left\{|a(\hat{\phi})|^{2}\right\}}{\int E\left\{|a(\hat{\phi})|^{2}\right\} d \hat{\phi}}
$$

is the normalized average power received from direction $\hat{\phi}$. In the case of a 2 -D multipath field, $\mathcal{P}(\hat{\phi})$ is known as the azimuth power distribution (APD) of the scatterers surrounding the receiver antenna region.

\section{2-D SCATTERING ENVIRONMENT}

Consider the situation where the signals arrive from the azimuthal plane only. Then the modal expansion of the plane wave $e^{i k \boldsymbol{x} . \hat{\boldsymbol{y}}}$ is $[6$, page 67]

$$
e^{i k \boldsymbol{x} \cdot \hat{\boldsymbol{y}}}=\sum_{n=-\infty}^{\infty} i^{n} J_{n}(k\|x\|) e^{i n\left(\phi_{x}-\phi_{y}\right)},
$$

where $J_{n}(x)$ is the integer order $n$ Bessel function, $\boldsymbol{x} \equiv\left(\|\boldsymbol{x}\|, \phi_{x}\right)$ and $\hat{\boldsymbol{y}} \equiv\left(1, \phi_{y}\right)$ in the polar coordinate system.

\subsection{Space-Time Cross Correlation (STCC)}

Substitution of (6) into (4) gives the space-time cross correlation of signals $s_{1}(t)$ and $s_{2}(t)$ for a 2-D scattering environment as

$$
R_{1,2}(\tau)=e^{i \omega_{c} \tau} \sum_{n=-\infty}^{\infty} i^{n} \alpha_{n} J_{n}(k\|\boldsymbol{u}(\tau)\|) e^{i n \phi_{u}},
$$

where $\boldsymbol{u}(\tau) \equiv\left(\|\boldsymbol{u}(\tau)\|, \phi_{u}\right)$ in polar coordinates and

$$
\alpha_{n}=\int_{0}^{2 \pi} \mathcal{P}(\phi) e^{-i n \phi} d \phi,
$$

where $\mathcal{P}(\phi)$ is the azimuth power distribution at the receiver. Note that the coefficients $\alpha_{n}$ characterize the scattering environment surrounding the receiver region. Closed form expressions for the scattering coefficients (8) were derived in [5] for several uni-modal azimuth power distributions.

Bessel functions $J_{n}(x)$ for $|n|>0$ exhibit a spatially high pass character $\left(J_{0}(x)\right.$ is spatially low pass), that is, $J_{n}(x)$ starts small and reaches to its maximum at arguments $x \approx O(n)$ before decaying slowly. Therefore, to compute the space-time cross correlation, one needs to compute only $2\lceil\pi e d / \lambda\rceil+1$ terms in the sum (7), where $d=\|\boldsymbol{u}(\tau)\|$, which gives a very good approximation to the true correlation [7].

\subsection{Space-Frequency Cross Spectrum (SFCS)}

The space-frequency cross spectrum is defined as

$$
S_{1,2}(\omega)=\mathcal{F}\left\{R_{1,2}(\tau)\right\}
$$

where $\mathcal{F}\{\cdot\}$ is the Fourier transform with respect to $\tau$. To evaluate $S_{1,2}(\omega)$, first we expand the term $J_{n}(k\|\boldsymbol{u}(\tau)\|) e^{i n \phi_{u}}$ in (7) as shown below. Let $\boldsymbol{x}=\boldsymbol{x}_{2}-\boldsymbol{x}_{1} \equiv\left(\|\boldsymbol{x}\|, \phi_{x}\right), \tau v \hat{\boldsymbol{v}} \equiv(\tau v, \gamma)$ and $\boldsymbol{u}(\tau) \equiv\left(\|\boldsymbol{u}(\tau)\|, \phi_{u}\right)$ in polar coordinates, $\theta_{x}=\gamma-\phi_{x}$ and maximum angular Doppler spread $\omega_{D}=v k$ (maximum Doppler frequency spread $f_{D}=v / \lambda$ ). Then, applying the summation theorem for Bessel functions $[8,8.530]$ on the argument of $J_{n}(k\|\boldsymbol{u}(\tau)\|)$, we obtain

$$
\begin{gathered}
J_{n}(k\|\boldsymbol{u}(\tau)\|) e^{i n \phi_{u}}=e^{i n \phi_{x}} \sum_{q=-\infty}^{\infty}(-1)^{q} J_{q}\left(\omega_{D} \tau\right) \times \\
J_{n+q}(k\|\boldsymbol{x}\|) e^{-i q \theta_{x}} .
\end{gathered}
$$

Substituting (10) in (7) and then taking the Fourier transform with respect to $\tau$ yields

$$
\begin{aligned}
S_{1,2}(\omega)= & \sum_{n=-\infty}^{\infty} \alpha_{n} e^{i n\left(\frac{\pi}{2}+\phi_{x}\right)} \sum_{q=-\infty}^{\infty}(-1)^{q} e^{-i q \theta_{x}} \times \\
& \mathcal{F}\left\{J_{q}\left(\omega_{D} \tau\right) e^{i \omega_{c} \tau}\right\} J_{n+q}(k\|\boldsymbol{x}\|) .
\end{aligned}
$$

The space-frequency cross spectrum $S_{1,2}(\omega)$ is centered on the carrier frequency and is zero outside the limits of $\omega_{c} \pm \omega_{D}$. Therefore we only consider the frequencies up to the maximal angular Doppler spread $\omega_{D}$. Then the Fourier transform $F_{q}(\omega)$ of $J_{q}\left(\omega_{D} \tau\right)$ for $\omega<$ $\omega_{D}$ is given by [9, page 66]

$$
F_{q}(\omega)= \begin{cases}G(\omega) \cos \left\{q \sin ^{-1}\left(\frac{\omega}{\omega_{D}}\right)\right\}, & q \geq 0 \\ (-1)^{|q|} G(\omega) \cos \left\{|q| \sin ^{-1}\left(\frac{\omega}{\omega_{D}}\right)\right\}, & q<0,\end{cases}
$$

where $G(\omega)=\left(\omega_{D}^{2}-\omega^{2}\right)^{-\frac{1}{2}}$. Substitution of $F_{q}(\omega)$ into (11) and using the frequency shifting theorem, we obtain the space-frequency cross spectrum

$$
S_{1,2}(\omega)=\sum_{n=-\infty}^{\infty} \alpha_{n} \beta_{n}, \text { where }
$$

$$
\beta_{n}=e^{i n\left(\frac{\pi}{2}+\phi_{x}\right)} \sum_{q=-\infty}^{\infty}(-1)^{q} e^{-i q \theta_{x}} F_{q}\left(\omega-\omega_{c}\right) J_{n+q}(k\|\boldsymbol{x}\|)
$$

and $\alpha_{n}$ as given in (8). Note that, as in the space-time cross correlation case, here we need to compute only $2\lceil\pi e\|\boldsymbol{x}\| / \lambda\rceil+1$ terms of the infinite sum associated with $\beta_{n}$ above. Furthermore, for a given antenna configuration and MU movement, $\beta_{n}$ can be precomputed for all angular frequencies interested. As shown in [5] closed form solutions for the scattering environment coefficients $\alpha_{n}$ exist for many commonly used scattering distributions. Therefore, the space-time cross correlation $R_{1,2}(\tau)$ and the space-frequency cross spectrum $S_{1,2}(\omega)$ can be computed for a wide range of scattering environments using (7) and (12). Table 1 summarizes scattering coefficients $\alpha_{n}$ for several commonly used uni-modal azimuth power distributions.

Both $R_{1,2}(\tau)$ and $S_{1,2}(\omega)$ are expressed in terms of MU antenna placement relative to a receiver origin, speed and the traveling direction of the MU, and the scattering environment coefficients, which captures the characteristics of the surrounding scattering environment at the MU. Therefore our expressions give an advantage over the existing expressions [1-3] as they do not depend on the scatterer arrangements (one-ring model), position of the MU relative to the transmitter (distance from the transmitter and the angle) and the antenna placement at the transmitter side. 


\begin{tabular}{|c|c|}
\hline Uni-modal APD & Scattering Coefficients $\left(\alpha_{n}\right)$ \\
\hline Uniform limited & $\exp \left(-i n \phi_{0}\right) \frac{\sin (n \Delta)}{n \Delta}$ \\
\hline Gaussian & $\exp \left(-i n \phi_{0}-n^{2} \sigma_{G}^{2} / 2\right)$ \\
\hline $\cos ^{2 p} \phi$ distributed field & $\exp \left(-i n \phi_{0}\right) \frac{\Gamma^{2}(p+1)}{\Gamma(p-n+1) \Gamma(p+n+1)}$ \\
\hline von-Mises & $\begin{aligned} \exp \left(-i n \phi_{0}\right) \frac{I_{-n}(\kappa)}{I_{0}(\kappa)} \\
I_{n}(\kappa): \text { modified Bessel function } \\
\\
\text { of the first kind. }\end{aligned}$ \\
\hline Laplacian & $\begin{array}{c}\exp \left(-i n \phi_{0}\right) \frac{\left(1-(-1)^{\lceil n / 2\rceil} \xi F_{n}\right)}{\left(1+\sigma_{L}^{2} n^{2} / 2\right)(1-\xi)} \\
F_{n}=1 \text { for } n \text { even, } F_{n}=n \sigma_{L} / \sqrt{2} \\
\text { for } n \text { odd and } \xi=\exp \left(\frac{-\pi}{\sigma_{L} \sqrt{2}}\right) .\end{array}$ \\
\hline
\end{tabular}

Table 1. Scattering Coefficients $\left(\alpha_{n}\right)$ for Uniform Limited, Gaussian, $\cos ^{2 p} \phi$, von-Mises and Laplacian azimuth power distributions

\subsection{Uni-modal Distributed Field within a Limited Spread}

Results in [1-3] have been based on the distribution of scatterers over the entire spatial horizon. However, using the method we presented in this paper, it is possible to explicitly account for the impact of limited spreads with an arbitrary distribution within the limited spread. For example, we consider a Laplacian distributed field within the limited spread $\left(-\theta_{0}, \theta_{0}\right)$. The truncated Laplacian distribution function is given by,

$$
\mathcal{P}(\phi)=\frac{K_{L}}{\sqrt{2} \sigma_{L}} e^{-\sqrt{2}\left|\phi-\phi_{0}\right| / \sigma_{L}}, \quad\left|\phi-\phi_{0}\right| \leq \theta_{0}, \quad\left|\theta_{0}\right| \leq \pi,
$$

where $K_{L}$ is the normalization constant, $\sigma_{L}$ is the standard deviation of the non-truncated distribution, which is related to the angular spread $(\sigma)$ at the receiver, and $\phi_{0}$ is the mean AOA. In this case, scattering coefficients

$$
\alpha_{n}=e^{-i n \phi_{0}} \frac{\left(1-\xi\left(\cos n \theta_{0}-\lambda_{n} \sin n \theta_{0}\right)\right)}{(1-\xi)\left(1+n^{2} \sigma_{L}^{2} / 2\right)},
$$

where $\lambda_{n}=n \sigma_{L} / \sqrt{2}$ and $\xi=e^{-\sqrt{2} \theta_{0} / \sigma_{L}}$. Fig.1 shows the magnitude of the space-time correlation function (7) for a Laplacian distributed field with mean AOA $\phi_{0}=60^{\circ}$ from broadside, limited spread $\theta_{0}=90^{\circ}$ around the mean AOA and angular spreads $\sigma=\left\{20^{\circ}, 10^{\circ}\right\}$, varying antenna separation $\left\|\boldsymbol{x}_{2}-\boldsymbol{x}_{1}\right\|$ and $\tau$. Here we assumed that two antennas are placed on the $x$-axis (i.e., $\phi_{x}=0$ ), the travelling direction of the mobile is $\gamma=30^{\circ}$ from end-fire of the receiver antennas and maximum Doppler frequency $f_{D}=\omega_{D} / 2 \pi=0.05$. In this case, the MU is traveling directly towards the strongest signal reception direction, which is the mean AOA of the distribution. Fig. 2 shows the corresponding spacefrequency cross spectrum for the Laplacian distributed field and receiver antenna separations $\left\|\boldsymbol{x}_{2}-\boldsymbol{x}_{1}\right\|=\{0.1 \lambda, 0.5 \lambda, \lambda\}$.
From Fig.1, it is observed that after $\tau=20$ time samples, spacetime cross correlation is insignificant $\left(\left|R_{1,2}(\tau)\right|<0.3\right)$ for both angular spreads when the receive antenna separation is small. Furthermore, for all values of $\tau$, the space-time cross correlation is negligible when the receiver antenna separation is larger than $0.75 \lambda$ and $1.5 \lambda$ for angular spreads $20^{\circ}$ and $10^{\circ}$, respectively. In general, we can observe that, $\left|R_{1,2}(\tau)\right|$ increases as the angular spread and antenna separation decreases and also with small number of time samples. From Fig. 2 it can be seen that space-frequency cross spectrum is less sensitive to antenna separation for both angular spreads when the MU is traveling directly towards the mean AOA. A similar observation is made when the MU is moving directly away from the mean AOA (i.e. $\gamma=210^{\circ}$ ) (simulation results are not provided here). Using $R_{1,2}(\tau)$ in (7) and $S_{1,2}(\|\boldsymbol{x}\|, \omega)$ in (12), it is evident that a detailed simulation study could be performed for various spatial scenarios and MU motions. Such a simulation study will reveal the impact of scattering environment, antenna spacing and MU motion on the performance of communication systems, and will be reported in a future publication.

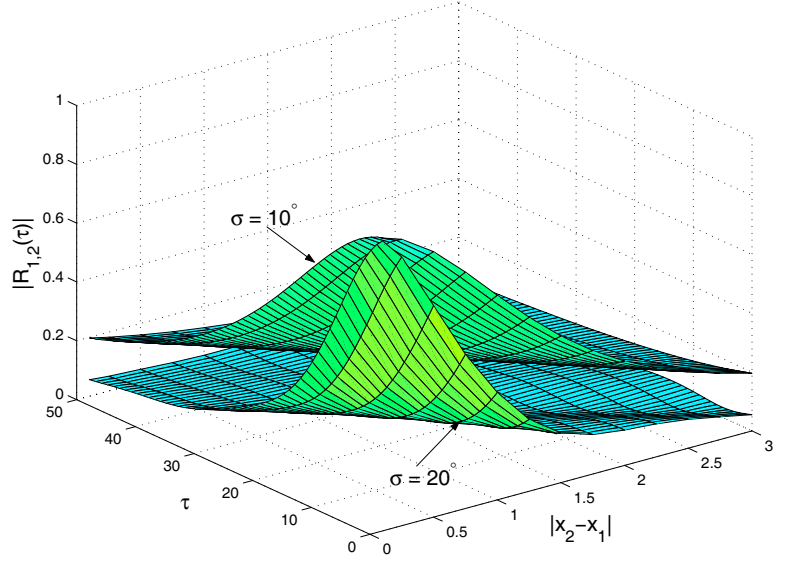

Fig. 1. Magnitude of the STCC function for $f_{D}=\omega_{D} / 2 \pi=0.05$, $\gamma=30^{\circ}$ and a Laplacian distributed field with mean $\mathrm{AOA}=60^{\circ}$ from broadside and angular spreads $\sigma=\left\{20^{\circ}, 10^{\circ}\right\}$.



Fig. 2. Magnitude of the SFCS function for $f_{D}=\omega_{D} / 2 \pi=0.05$, $\gamma=30^{\circ},\left\|\boldsymbol{x}_{2}-\boldsymbol{x}_{1}\right\|=\{0.1 \lambda, 0.5 \lambda, \lambda\}$ and a Laplacian distributed field with mean $\mathrm{AOA}=60^{\circ}$ from broadside and angular spreads $\sigma=$ $\left\{20^{\circ}, 10^{\circ}\right\}$ 


\subsection{Multi-modal Distributed Field}

A multi-modal azimuth power distribution arises when there are two or more strong multipaths exist in a fading channel. This may be the result of multiple remote macroscopic scattering regions, for instance. A multi-modal distribution can be constructed from a mixture of uni-modal distributions. For example, here we construct a multi-modal distribution from a mixture of von-Mises distributions, where each mode (strong multipath) is represented by a mixture component with a mean value $\phi_{\ell}$ and a concentration parameter $\kappa_{\ell}$ :

$$
\mathcal{P}(\phi)=\frac{1}{2 \pi} \sum_{\ell=1}^{M} \frac{\gamma_{\ell}}{I_{0}\left(\kappa_{\ell}\right)} e^{\kappa_{\ell} \cos \left(\phi-\phi_{\ell}\right)}, \quad \phi \in[-\pi, \pi),
$$

with $\sum_{\ell=1}^{M} \gamma_{\ell}=1$, where $M$ is the number of modes and $\gamma_{\ell}$ is the mixing coefficient which is independent of $\phi$. For $M=1$, the distribution becomes uni-modal von-Mises. Using [8, 3.937], scattering coefficients

$$
\begin{aligned}
\alpha_{n} & =\sum_{\ell=1}^{M} \frac{\gamma_{\ell}}{2 \pi I_{0}\left(\kappa_{\ell}\right)} \int_{0}^{2 \pi} e^{\kappa_{\ell} \cos \left(\phi-\phi_{\ell}\right)} e^{i n \phi} d \phi, \\
& =\sum_{\ell=1}^{M} \frac{\gamma_{\ell} I_{-n}\left(\kappa_{\ell}\right)}{I_{0}\left(\kappa_{\ell}\right)} e^{-i n \phi_{\ell}} .
\end{aligned}
$$

We now study the correlation effects due to uni-modal and bimodal distributions at the receiver aperture. Figure 3(a) and 3(b) depict the bi-modal von-Mises distributions for mean AOA $\phi_{0}=0^{\circ}$ and non-isotropic parameters (concentration parameters) $\kappa_{1}=\kappa_{2}=$ 200. In Figure 3(a), modes are located at $\phi_{1}=-25^{\circ}$ and $\phi_{2}=25^{\circ}$, and in Figure 3(b), modes are located at $\phi_{1}=-15^{\circ}$ and $\phi_{2}=15^{\circ}$. For both cases we set mixture coefficients $\gamma_{1}=\gamma_{2}=0.5$. In the first case, the angular spread $\sigma$ at the receiver is $25^{\circ}$ and in the second case it is $15^{\circ}$. Also shown in Figure 3(a) and 3(b) are the uni-modal von-Mises distributions with mean AOA $\phi_{0}=0^{\circ}$ and the receiver angular spread $25^{\circ}(\kappa=6)$ and $15^{\circ}(\kappa=14)$, respectively. Figure $3(\mathrm{c})$ and $3(\mathrm{~d})$ show the corresponding spatial correlation between two receiver antennas against the spatial separation for $\tau=0$. Scattering coefficients $\alpha_{n}$ and correlation coefficients $R_{1,2}(0)$ are calculated using (13) and (7), respectively. From Figure 3(c) and 3(d) we can observe that bi-modal distributions give slightly less spatial correlation than uni-modal distributions for small antenna separations. However, at large antenna separations $\left(\left\|\boldsymbol{x}_{2}-\boldsymbol{x}_{1}\right\|>\lambda\right)$, the spatial correlation results from bi-modal distributions is significant compared to that of uni-modal distributions.

\section{CONCLUSION}

We have derived expressions for space-time cross correlation and space-frequency cross spectrum between signals at two antennas on a mobile unit for arbitrary scattering environments. Unlike previously derived space-time/frequency cross correlation/spectrum functions, current expressions only dependent on the antenna placement at the MU, speed and the traveling direction of the MU and the scattering environment coefficients. These new expressions can be used to obtain new insights about the role of space between antenna elements, antenna placement, MU motion, mean AOA and angular spread of surrounding APD on the performance of MIMO systems and will be reported in a future publication. Also, the analysis presented in this paper can be extended to frequency selective fading channels by considering a propagation delay on waves arriving in direction $\hat{\phi}$.
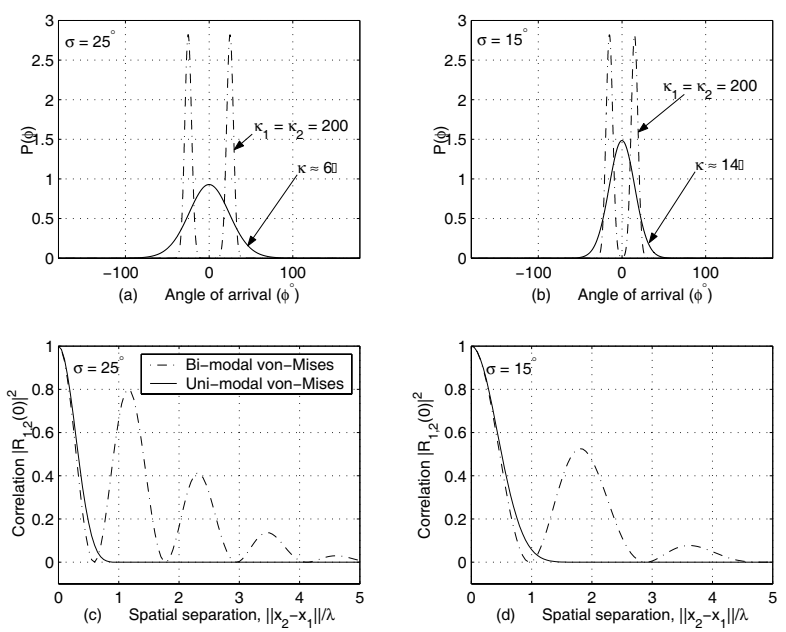

Fig. 3. Comparison of uni-modal and bi-modal von-Mises distributions.

\section{REFERENCES}

[1] T. A. Chen, M. P. Fitz, W. Y. Kuo, M. D. Zoltowski, and J. H. Grimm, "A spacetime model for frequency nonselective rayleigh fading channels with applications to space-time modems," IEEE Journal on Selected Areas in Communications, vol. 18, pp. 1175-1190, July 2000.

[2] D. B. Smith and T. D. Abhayapala, "Generalised space-time model for rayleigh fading channels with non-isotropic scatterer distribution," IEE Electronics Letters, vol. 39, no. 21, pp. 15411543, Oct. 2003.

[3] A. Abdi and M. Kaveh, "A space-time correlation model for multielement antenna systems in mobile fading channels," IEEE Journal on Selected Areas in Communications, vol. 20, pp. 550560, May 2002.

[4] A. Abdi, J. A. Barger, and M. Kaveh, "A parametric model for the distribution of the angle of arrival and the associated correlation function and power spectrum at the mobile station," IEEE Trans. Vehic. Technol., vol. 51, no. 3, pp. 425-434, May 2002.

[5] P. D. Teal, T. D. Abhayapala, and R. A. Kennedy, "Spatial correlation for general distributions of scatterers," IEEE Signal Processing Letters, vol. 9, no. 10, pp. 305-308, Oct. 2002.

[6] D. Colton and R. Kress, Inverse Acoustic and Electromagnetic Scattering Theory, vol. 93, Springer, New York, 2 edition, 1998.

[7] H. M. Jones, R. A. Kennedy, and T. D. Abhayapala, "On dimensionality of multipath fields: Spatial extent and richness," in Proc. IEEE Int. Conf. Acoust., Speech, Signal Processing, ICASSP'2002, Orlando, Florida, May 2002, vol. 3, pp. $2837-$ 2840.

[8] I. S. Gradshteyn and I. M. Ryzhik, Table of Integrals, Series and Products, Academic Press, London, 6 edition, 2000.

[9] F. Oberhettinger, Tables of Fourier transforms and Fourier transforms of distributions, Springer-Verlag, Berlin, 2 edition, 1990. 